\title{
Medizinstudium: mehr als nur Bücher
}

Sarah Schroth

Korrespondenz: Sarah Schroth Weyermannstrasse 44 CH-3008 Bern sarah.schroth[at] students.unibe.ch

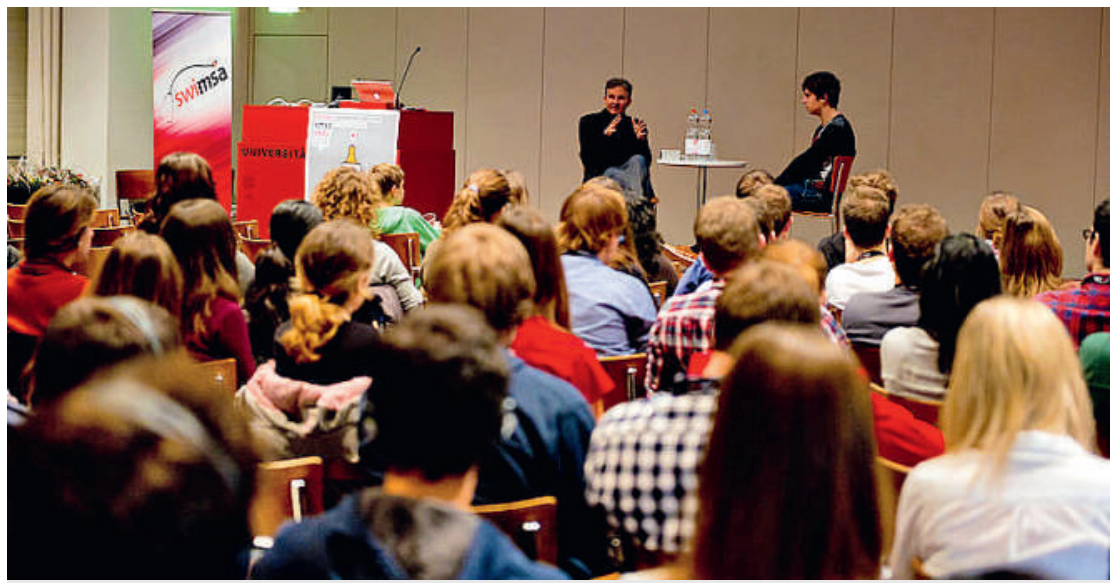

220 Medizinstudenten aus der ganzen Schweiz besuchten die diesjährige «Swiss Medical Students' Convention» in Basel. Neben lehrreichen Vorträgen gab es zahlreiche Workshops und viel Erfahrungsaustausch.
Zweimal im Jahr findet an einer der Medizinfakultäten der Schweiz die Swiss Medical Students' Convention (SMSC) statt. Was im Jahr 2006 mit knapp 16 Teilnehmern begonnen hat, ist heute das grösste und wichtigste interkantonale Zusammentreffen von Schweizer Medizinstudenten. Diesen Herbst besuchten knapp 220 Studenten die 12. SMSC in Basel zum Thema «Pediatrics - Children are our Future».

\section{Ursprung der SMSC}

Das Konzept der SMSC ist international schon länger bekannt. Kongresse für Medizinstudenten mit Workshops, Diskussionen und Vorträgen zu einem bestimmten Themenbereich finden seit vielen Jahren in fast allen Ländern der Welt regelmässig statt. In Amerika werden Kongresse mit mehr als 1000 Studenten erfolgreich organisiert. In Europa sind Holland und Deutschland mit Teilnehmerzahlen um die 600 sehr aktiv und engagiert. Die Kongresse finden zum Teil auf nationaler Ebene und teilweise länderübergreifend statt und bieten dadurch eine ideale Gelegenheit, um den nationalen und internationalen Austausch zwischen Medizinstudenten zu erhalten und zu fördern. In der Schweiz ist die SMSC der offizielle Kongress der swimsa (Swiss Medical Students' Association), die den ersten SMSC 2005 auch in der Schweiz lancieren konnten.

\section{Organisation der SMSC}

Verantwortlich für Planung und Ablauf der Kongresse ist das Organisationskomitee, bestehend aus cirka

\section{Les études de médecine vont bien au-delà de la simple formation}

Le Swiss Medical Students' Convention (SMSC) est un congrès organisé en Suisse depuis 2005 par et pour des étudiants en médecine. Deux fois par an, la Swiss Medical Students' Association (swimsa) met sur pied cette manifestation de deux jours dans l'une des facultés de médecine suisses. A ses débuts à Berne en 2005, celle-ci regroupait à peine 16 étudiants alors qu'elle en compte plus de $\mathbf{2 0 0}$ aujourd'hui. En plus de conférences intéressantes et de workshops interactifs, une table ronde est également organisée avec des invités triés sur le volet pour traiter de questions d'actualité. Dans le cadre de ce congrès, le «Project Fair» permet aussi aux différentes organisations de proposer des stands d'information sur de nombreux projets, tels l'Hôpital des nounours ou le projet d'éducation sexuelle «Achtung Liebe». Le dernier congrès de la SMSC qui s'est déroulé à Bâle début novembre sur le thème «Pediatrics - Children are our Future» a rencontré un immense succès dans une ambiance détendue.

10 Medizinstudenten der Gastgeberfakultät. Diese gestalten nicht nur das Fachprogramm zum entsprechenden Thema, sondern stellen auch mit einem verhältnismässig bescheidenen Budget das Rahmenprogramm inklusive Essen, Übernachtung und Entertainment für alle Teilnehmer zusammen. Eine riesige Leistung für eine Handvoll Studierender, wenn man bedenkt, dass das Studium mit allen Vorlesungen, Praktika und Prüfungen derweilen kein Erbarmen zeigt.

\section{SMSC Basel - November 2011}

Doch auch dieses Jahr hat sich die harte Arbeit, mit wochenlanger Sponsorenjagd und schlaflosen Nächten, zweifellos gelohnt. Der Herbstkongress, der Anfangs November in Basel stattfand, liess keine Wünsche offen. Das Kinderspiel «Schokolade auspacken» 


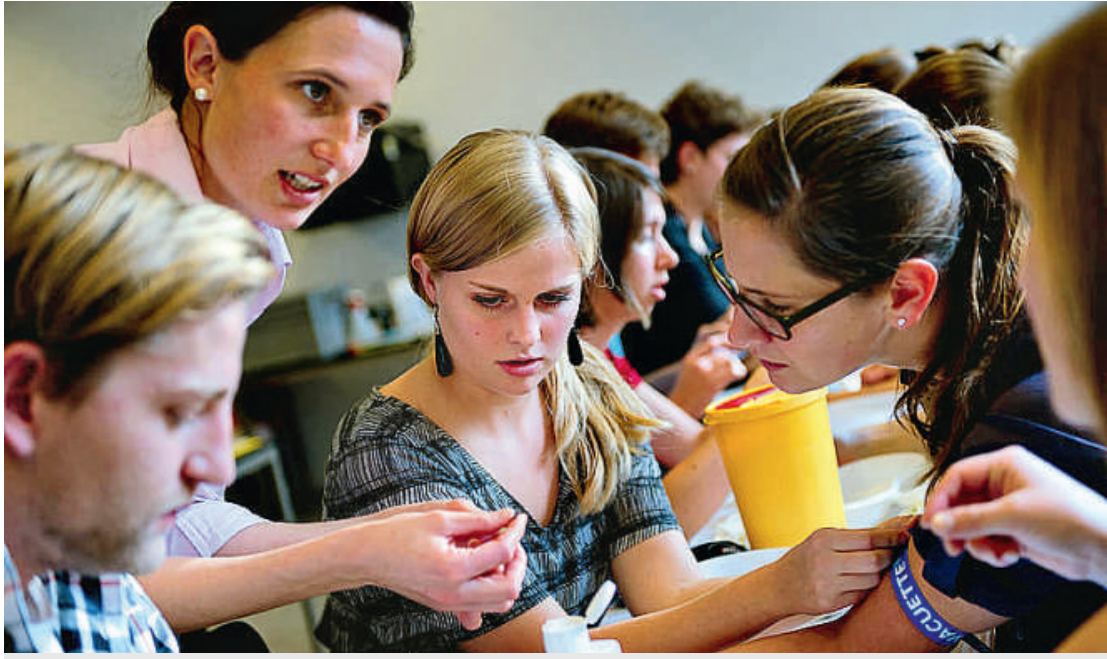

Einmal Blut abnehmen bitte - aber richtig! Wie das geht, wurde in einem der vielen Workshops gezeigt und ausprobiert.

gestaltete sich zum perfekten Einstieg und wurde durch den Besuch des Spitalclowns Dr. Ahoi der Theodora Stiftung mit viel Witz und Lachen komplettiert.

Am Samstag wurde das SMSC-Wochenende mit einem Vortrag zum Thema «Schwieriges Kind, schwieriger Doktor - der Dialog zwischen den Generationen» offiziell eröffnet. Der Referent, PD Dr. med. Alain Di Gallo, Chefarzt des Kinder- und Jugendpsychiatrischen Dienstes der Kantonalen Psychiatrischen Dienste Basel-Landschaft, faszinierte neben einem allgemeinen Überblick zu seinem Beruf und seiner Arbeitsweise durch ein berührendes Live-Gespräch mit einem seiner langjährigen Patienten. Die Begeisterung und Freude, die Dr. Di Gallo offensichtlich an seinem Beruf hat, zog alle in ihren Bann.

Einen anderen Blickwinkel auf die Medizin bot der Vortrag mit dem Titel «Generika - Ein aktueller Überblick aus Sicht des Schweizer Marktführers». Ein Vertreter der «Mepha» lieferte einen spannenden Einblick in das kontrovers diskutierte und hochrelevante Thema. Eine gelungene Präsentation $\mathrm{zu}$ einem Thema, das zwar nicht im Staatsexamen, aber im Rahmen der aktuellen Sparmassnahmen in der späteren Praxis sehr präsent sein wird.

Genauso wichtig für die zukünftige Ärzteschaft sind der richtige Umgang mit den kleinen Patienten und natürlich die richtige Diagnostik. Das lehrreiche Referat von Dr. med. Urs Frey, Chefarzt der Pädiatrie des Universitäts-Kinderspitals beider Basel UKBB, über «Atemnot beim Kind und Säugling», lieferte einen gelungenen Überblick - von Asthma über Pneumonie bis zur Zyanose. Dass B-Streptokokken und Staphylococcus aureus zwei der häufigsten Erreger von Pneumonien bei Neugeborenen sind und kurzwirksame ß2-Mimetika die erste Massnahme bei akutem Asthmaanfall, werden spätestens seit dem SMSC 214 Studierende der Schweiz nicht mehr so schnell vergessen.

Da Erfahrungsaustausch und Diskussionen seit eh und je ein Kernbestandteil der SMSC sind, durfte auch dieses Jahr die traditionelle Podiumsdiskussion nicht fehlen. Das diesjährige Thema «Präimplantationsdiagnostik» entwickelte sich zu einer hitzigen und spannenden Debatte zwischen Gegnern und Befürwortern. Neben einem betroffenen Ehepaar waren vier Experten und Interessenvertreter mit von der Partie und lösten bei vielen Studenten neue Überlegungen und Denkanstösse aus.

\section{Workshops}

Ein besonderes Highlight stellt jedes Jahr das Angebot einer Vielzahl interaktiver Workshops dar, für die sich die Teilnehmer vorab anmelden können. Dieses Jahr wurden unter anderem Workshops von JDMT Medical Services, der Hebammenvereinigung Basel, Unilabs und der Schweizer Vereinigung für Homöopathie angeboten. Wie untersuche ich ein Neugeborenes? Was macht eigentlich eine Hebamme? - alles Fragen, die in den Workshops interaktiv behandelt wurden. An zwei Nachmittagen wurden Puppen «reanimiert», Blutproben gezapft und Laborresultate analysiert. Das Fazit einer begeisterten Workshop-Teilnehmerin: «Hier ist Lernen keine mühsame Pflicht, sondern macht richtig Spass und Freude. Das motiviert mich dazu, meine Lerntechnik auch weiterhin kreativ und spielerisch zu gestalten.»

\section{Projekt Fair}

Die SMSC bietet zudem eine exzellente Plattform, um auf diverse Projekte, Verbände und Initiativen aufmerksam zu machen. Unilabs, SAMK (Kompetenzzentrum für Militär- und Katastrophenmedizin), Interessenverband für Schweizer Ärzte und Ärztinnen, Stiftung Theodora, Teddybär Spital und das Aufklärungsprojekt Achtung Liebe sind eine kleine Auswahl der zahlreichen Stände, an denen man sich auf der SMSC informieren konnte. Für Studenten bietet die SMSC so eine ideale Gelegenheit, interessante Kontakte zu knüpfen und die Medizin ausserhalb der Bibliotheken und Hörsäle zu entdecken.

\section{Fazit der SMSC}

«Medizinstudium bedeutet mehr als nur Bücher!» Mit diesem prägnanten Schlusssatz beendete Alexandre Moser, Präsident der swimsa, den 12. und einen der bisher grössten Medizinstudentenkongresse der Schweiz. Auch dieses Jahr hat die SMSC bewiesen: Kreativ, interaktiv und kommunikativ gestaltete Medizin macht einfach Spass, sehr viel Spass sogar! Die nächste SMSC wird im März 2012 stattfinden. Dann ist in Lausanne «Action» angesagt, denn das geplante Thema «Katastrophenmedizin» wird mit Sicherheit einige Pulse in die Höhe treiben. Lassen wir uns überraschen.

Um die SMSC weiterhin durchführen zu können und der wachsenden Nachfrage gerecht zu werden, suchen wir weiterhin aktive Mitglieder, Helfer und Sponsoren. Mehr Informationen zum SMSC finden sich unter www.swimsa.ch/smsc. Für Fragen steht die swimsa jederzeit zur Verfügung: contact[at]swimsa.ch 